\title{
Dual Branch Transmit Switch-and-Stay Diversity for Underlay Cognitive Networks
}

\author{
Mostafa Sayed*, Mohamed Abdallah**, Mohamed-Slim Alouini ${ }^{\S}$, Khalid A. Qaraqe** \\ *Varkon Semiconductors, Maadi, Cairo, Egypt \\ Email: mostafa_sayed@varkonsemi.com \\ **Electrical and Computer Engineering, Texas A\&M University at Qatar, PO Box 23874, Doha, Qatar \\ Email: mohamed.abdallah, khalid.qaraqe@qatar.tamu.edu \\ $\S$ Electrical Engineering Program, KAUST, Thuwal, Saudi Arabia \\ Email: mohamed.alouini@kaust.edu.sa
}

\begin{abstract}
In this paper, we study applying dual branch transmit switch-and-stay combining (SSC) technique for underlay cognitive radio (UCR) networks. In UCR, the secondary user is allowed to share the spectrum with the primary (licensed) user under the condition that interference at the primary receiver is below a predetermined threshold. Assuming binary phaseshift keying (BPSK) modulation and Rayleigh fading channels, we develop a closed form expression for the average bit error rate (BER) of the secondary link as a function of the switching threshold. We then find a closed form expression for the optimal switching threshold in the sense of minimizing the average BER. For the sake of comparison we derive an expression for the average BER of the dual branch transmit selection combining (SC) technique. We finally investigate the effect of correlation between secondary and interference channels on the average BER and the associated optimal switching threshold.
\end{abstract}

Index Terms-Transmit selection diversity, Transmit switched diversity, Underlay cognitive radio, Multiple antenna systems.

\section{INTRODUCTION}

Underlay cognitive radio (UCR) has been introduced recently to serve the increasing demand for spectrum by allowing secondary users to share the spectrum of primary users under the constraint of maximum interference allowed at the primary receiver. In order to meet the interference constraint, a power adaptation scheme is applied at the secondary transmitter by adjusting its power according to the channel gain between the secondary transmitter and the primary receiver. However, this power adaptation can affect the performance of the secondary link especially if the channel gain between the secondary transmitter to the primary receiver is higher than that between the secondary transmitter and secondary receiver. One way to alleviate such problem is employing multiple antennas at the transmitter whereby the secondary transmitter can perform transmit selection techniques to improve the performance of the secondary link while maintaining the interference constraint [6]. For example, the authors in [6] have investigated the best transmit antenna selection technique where the secondary transmitter selects the best antenna that achieves the best performance in terms of capacity. However, such technique

This work is supported by Qatar National Research Fund (QNRF) grant through National Priority Research Program (NPRP) No. 29-6-7-4. QNRF is an initiative of Qatar Foundation.

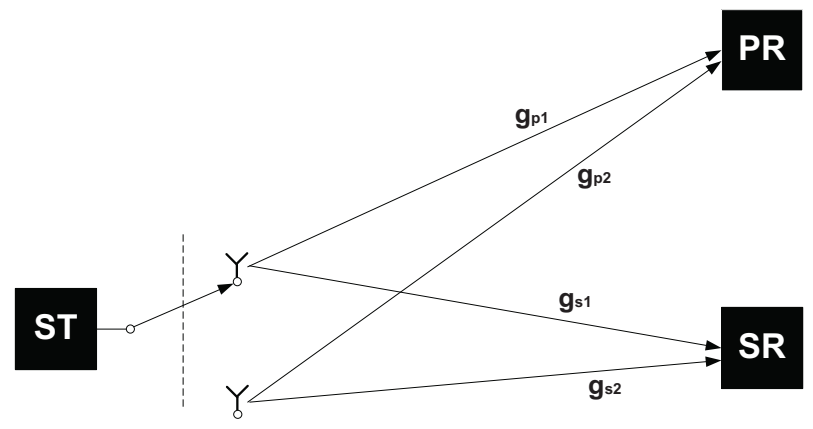

Fig. 1. System model for transmit SSC applied in an UCR network.

suffers from the disadvantage of continuous monitoring of all transmit diversity branches by the receivers which is then fedback to the secondary transmitter. Switched diversity techniques have been proved useful in providing less complex diversity technique on the expense of acceptable performance loss. In particular, switch-and-stay combining (SSC) technique for dual-branch antennas has been investigated in [2], [3], [4], [5]. For dual-branch transmit SSC technique, the transmitter switches to the other antenna when the received signal-tonoise ratio (SNR) of the current antenna falls below a certain threshold. The transmitter stays with this antenna independent of whether its received SNR is above or below the threshold.

In this paper, we study the performance of the dual branch transmit SSC technique for UCR systems under a peak interference constraint allowed at the primary receiver. In particular, we derive an expression for the average bit error rate (BER) in terms of the switching threshold. We also get an expression for the optimal threshold that minimizes the average BER at the secondary receiver. In order to evaluate the performance of the transmit SSC technique, we derive an expression for the average BER of the dual branch transmit selection combining (SC) technique for the sake of comparing the performance of the two techniques. Finally, we study the effect of correlation between the interference and the secondary channels on the average BER of the secondary link for the dual branch transmit SSC technique.

The remainder of this paper is organized as follows. In the 
next section, we present the system model. In Section III, we analyze the performance of dual branch transmit SSC showing how to calculate the optimal switching threshold. In Section $\mathrm{IV}$, we analyze the performance of dual branch transmit SC and provide performance comparison with the dual branch transmit SSC technique. Fading correlation between the secondary and interference channels is studied in Section V and some conclusions are presented in Section VI.

\section{SySTEM MODEL}

In this paper, we consider the system model shown in Fig. 1 wherein the secondary transmitter (ST) has two antennas while the primary receiver (PR) and the secondary receiver (SR) have single antennas. We assume the channels involved in communication are modeled as block Rayleigh fading channels. The channel gain between the $i$-th antenna of the secondary transmitter and the primary receiver (Interference Channel) is denoted by $g_{p_{i}}, i \in\{1,2\}$, and that between the $i$-th antenna of the secondary transmitter and secondary receiver (Secondary Channel) is denoted by $g_{s_{i}}, i \in\{1,2\}$. The channel gains $g_{p_{i}}$ and $g_{s_{i}}$ are independent and identicallydistributed (i.i.d) exponential random variables with mean values of $m_{p}$ and $m_{s}$, respectively. We assume independent transmit diversity branches i.e. there is no correlation between $g_{i}$ and $g_{k}$ for $i \neq k$. The secondary transmitter is assumed to have full knowledge of the channel state information (CSI) between the secondary transmitter and the primary and secondary receivers for the currently transmitting antenna via infiniteresolution error-free feedback channels. Let $Q$ denotes the maximum interference power allowed at the primary receiver caused by the secondary transmitter and let $p_{i}$ denotes the power transmitted by the secondary transmitter using the $i$-th antenna. In order to meet the peak interference constraint, the secondary transmitter applies a power adaptation scheme as follows,

$$
p_{i}=\frac{Q}{g_{p_{i}}} .
$$

Let $\gamma_{i}$ denotes the the secondary receiver SNR that corresponds to the $i$-th transmit diversity branch. Assuming Gaussian noise with zero mean and variance $N_{o} B$ where $B$ is the transmission bandwidth, $\gamma_{i}$ is given as follows,

$$
\gamma_{i}=\frac{p_{i} g_{s_{i}}}{N_{o} B}=\frac{Q}{N_{o} B} \frac{g_{s_{i}}}{g_{p_{i}}} .
$$

\section{Dual BRANCh Transmit SSC For UCR Networks}

Let $\gamma_{s s c}$ denotes the secondary receiver SNR with transmit SSC technique applied at the secondary transmitter and $\gamma_{T}$ denotes the predetermined switching threshold. In this section, we drive the probability density function (PDF), $f_{\gamma_{s s c}}\left(\gamma_{s s c}\right)$, and the moment generating function( MGF), $M_{\gamma_{s s c}}(s)$, of $\gamma_{s s c}$.

\section{A. The PDF of The Received SNR}

The PDF of $\gamma_{i}$ can be expressed as follows, [7].

$$
f_{\gamma}(\gamma)=\frac{\alpha}{(\gamma+\alpha)^{2}}
$$

where $\alpha=\frac{Q}{N_{o} B} \frac{m_{s}}{m_{p}}$.

The the cummulative density function (CDF) of $\gamma_{i}$ can be found by integrating its PDF as follows,

$$
F_{\gamma}(\gamma)=\int_{0}^{\gamma} f_{\gamma}(u) d u=\frac{\gamma}{\gamma+\alpha}
$$

Using the result obtained in [2], the PDF of $\gamma_{s s c}$ can be written in terms of the CDF, $F_{\gamma}(\gamma)$, and the PDF, $f_{\gamma}(\gamma)$, of the secondary receiver SNR, that corresponds to a singleantenna transmitter, as follows,

$$
f_{\gamma_{s c c}}\left(\gamma_{s s c}\right)= \begin{cases}F_{\gamma}\left(\gamma_{T}\right) f_{\gamma}\left(\gamma_{s s c}\right) & \gamma_{s s c}<\gamma_{T} \\ \left(1+F_{\gamma}\left(\gamma_{T}\right)\right) f_{\gamma}\left(\gamma_{s s c}\right) & \gamma_{s s c} \geq \gamma_{T}\end{cases}
$$

By substituting (3) and (4) in (5) the PDF of $\gamma_{s s c}$ can expressed as follows,

$$
f_{\gamma_{s s c}}\left(\gamma_{s s c}\right)= \begin{cases}A \frac{\alpha}{(\gamma+\alpha)^{2}}, & \gamma_{s s c}<\gamma_{T} \\ (1+A) \frac{\alpha}{(\gamma+\alpha)^{2}}, & \gamma_{s s c} \geq \gamma_{T}\end{cases}
$$

where $A=\frac{\gamma_{T}}{\gamma_{T}+\alpha}$.

\section{B. The MGF of The Received SNR}

The MGF of $\gamma_{i}$ is defined by

$$
M_{\gamma}(s)=\int_{0}^{\infty} \mathrm{e}^{\mathrm{s} \gamma} \mathrm{f}_{\gamma}(\gamma) \mathrm{d} \gamma=\int_{0}^{\infty} \frac{\alpha \mathrm{e}^{\mathrm{s} \gamma}}{(\gamma+\alpha)^{2}} \mathrm{~d} \gamma
$$

By replacing $s$ by $-p$, the MGF can be expressed as follows,

$$
M_{\gamma}(-p)=1+p \alpha \mathrm{e}^{\mathrm{p} \alpha} \operatorname{Ei}(-\mathrm{p} \alpha), \quad \mathrm{p} \geq 0,
$$

where $\operatorname{Ei}(\mathrm{x})=\int_{-\infty}^{\mathrm{x}} \frac{\mathrm{e}^{\mathrm{t}}}{\mathrm{t}} \mathrm{dt}$ is the exponential integral function [8]. Using the result obtained in [2], the MGF of $\gamma_{s s c}$ can be written in terms of the MGF, $M_{\gamma}(s)$, of the secondary receiver SNR as follows,

$$
M_{\gamma_{s s c}}(s)=F_{\gamma}\left(\gamma_{T}\right) M_{\gamma}(s)+\int_{\gamma_{T}}^{\infty} \mathrm{e}^{\mathrm{s} \gamma_{\mathrm{ssc}}} \mathrm{f}_{\gamma}\left(\gamma_{\mathrm{ssc}}\right) \mathrm{d} \gamma_{\mathrm{ssc}} .
$$

\section{Average BER for The Secondary Receiver}

Assuming BPSK transmission and using the MGF-based approach for the evaluation of the average BER of systems operating over fading channels [2], the average BER for a BPSK signal over a flat Rayleigh fading channel can be expressed in terms of the MGF of the received SNR as follows,

$$
P_{b}(E)=\frac{1}{\pi} \int_{0}^{\frac{\pi}{2}} M_{\gamma_{s s c}}\left(\frac{-1}{\sin ^{2}(\phi)}\right) d \phi
$$

which can be expressed using (9) as follows, 


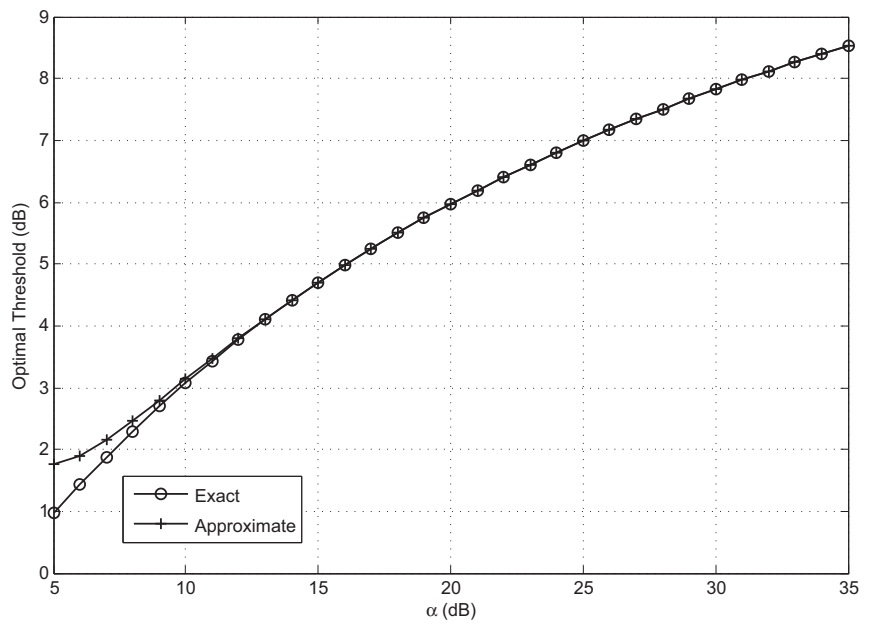

Fig. 2. The optimal switching threshold in $\mathrm{dB}$ of dual branch transmit SSC technique applied in an UCR network versus $\alpha$ in $\mathrm{dB}$. The modulation scheme is BPSK. The fading channel is a flat Rayleigh channel.

$$
\begin{aligned}
P_{b}(E)= & \frac{1}{\pi} \int_{0}^{\frac{\pi}{2}} F_{\gamma}\left(\gamma_{T}\right) M_{\gamma}\left(\frac{-1}{\sin ^{2}(\phi)}\right) d \phi \\
& +\frac{1}{\pi} \int_{0}^{\frac{\pi}{2}}\left[\int_{\gamma_{T}}^{\infty} f_{\gamma}\left(\gamma_{s s c}\right) \mathrm{e}^{-\gamma_{\mathrm{ssc}} / \sin ^{2}(\phi)} \mathrm{d} \gamma_{\mathrm{ssc}}\right] d \phi
\end{aligned}
$$

By substituting (3), (4) and (8) in (11), the average BER can be expressed as a function of $\alpha$ and the switching threshold $\gamma_{T}$ as follows,

$$
P_{b}\left(E ; \alpha, \gamma_{T}\right)=\frac{A}{2}+\frac{1}{\pi}\left[A\left(\frac{\alpha}{\gamma_{T}} I_{1}+I_{2}\right)+I_{3}\right],
$$

where the integrals $I_{1}, I_{2}$ and $I_{3}$ have the following closedform expressions

$$
\begin{gathered}
I_{1}=\int_{0}^{\pi / 2} \mathrm{e}^{-\left(\frac{\gamma_{\mathrm{T}}}{\sin ^{2}(\phi)}\right)} \mathrm{d} \phi=\frac{\pi}{2} \operatorname{erfc}\left(\sqrt{\gamma_{\mathrm{T}}}\right), \\
I_{2}=\int_{0}^{\pi / 2}\left(\frac{\alpha}{\sin ^{2}(\phi)}\right) \mathrm{e}^{\left(\frac{\alpha}{\sin ^{2}(\phi)}\right)} \operatorname{Ei}\left(-\frac{\alpha}{\sin ^{2}(\phi)}\right) \mathrm{d} \phi \\
I_{3}=\int_{0}^{\pi / 2}\left(\frac{\alpha}{\sin ^{2}(\phi)}\right) \mathrm{e}^{\left(\frac{\alpha}{\sin ^{2}(\phi)}\right)} \\
\\
\times \operatorname{Ei}\left(-\frac{\alpha}{\sin ^{2}(\phi)}-\frac{\gamma_{\mathrm{T}}}{\sin ^{2}(\phi)}\right) \mathrm{d} \phi .
\end{gathered}
$$

Using the infinite series expansion of $\operatorname{Ei}(x)$ [8, Eq. (8.215)],

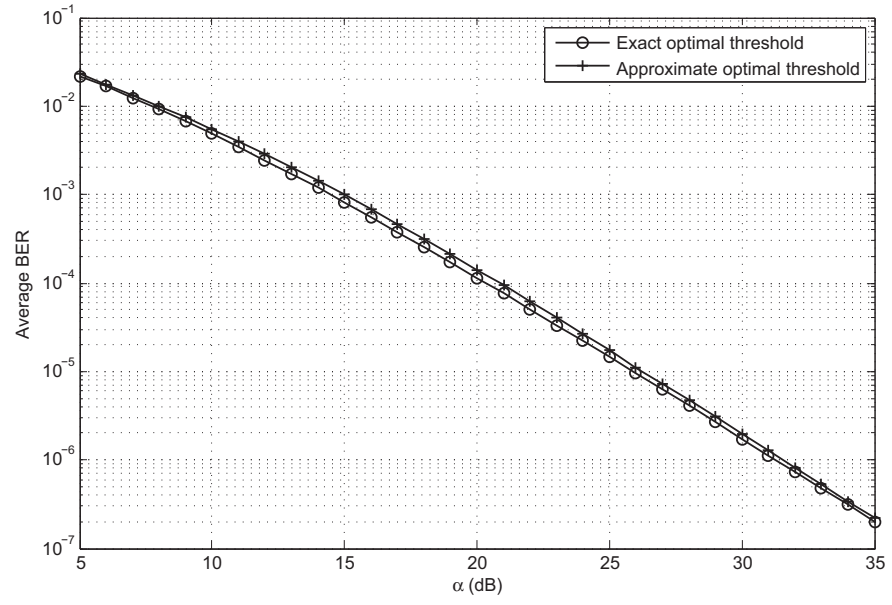

Fig. 3. Average BER of the dual branch transmit SSC in an UCR network for both exact and approximate expressions of the optimal switching threshold versus $\alpha$ in $\mathrm{dB}$ for BPSK transmission over flat Rayleigh channel.

$I_{2}$ can be equivalently represented by

$$
I_{2}=\frac{\pi}{2} \sum_{k=0}^{\infty}(-1)^{k+1} \frac{k !}{\alpha^{k}} \frac{(2 k-1) ! !}{(2 k) ! !} .
$$

Likewise, $I_{3}$ can be represented as

$$
\begin{aligned}
I_{3}= & \frac{\sqrt{\pi} \alpha}{2} \sum_{k=0}^{\infty}(-1)^{k+1} \frac{k !}{\left(\alpha+\gamma_{T}\right)^{k+1}} \\
& \times \frac{\mathrm{e}^{-\gamma_{\mathrm{T}} / 2}}{\gamma_{T}^{-(2 k-1) / 4}} W_{\frac{-(2 k+1)}{4}, \frac{-(2 k+1)}{4}}\left(\gamma_{T}\right),
\end{aligned}
$$

where $W_{\lambda, \mu}(z)$ is the Whittaker functions defined in [8, Eq. (9.220.4)].

Similarly, the average BER at the secondary receiver for a single-antenna transmitter can be calculated as follows,

$$
P_{b}(E ; \alpha)=\frac{1}{2}+\frac{1}{\pi} I_{2} .
$$

This expression is useful for comparing the results obtained for the transmit SSC case with the no diversity case in order to show the diversity gain of the transmit SSC technique.

\section{Optimal Switching Threshold}

The optimal switching threshold $\gamma_{T}^{*}$ that minimizes the average BER can be found by setting the first derivative of the average BER to zero and is given by [2],

$$
\gamma_{T}^{*}=\frac{1}{2}\left[Q^{-1}\left(\frac{1}{\pi} \int_{0}^{\pi / 2} M_{\gamma}\left(-\frac{1}{\sin ^{2}(\phi)}\right) d \phi\right)\right]^{2},
$$

where $Q^{-1}($.$) denotes the inverse Gaussian Q$-function. Substituting (8) in (19) yields 


$$
\gamma_{T}^{*}=\frac{1}{2}\left[Q^{-1}\left(\frac{1}{2}+\frac{1}{\pi} I_{2}\right)\right]^{2}
$$

and by using (16), the optimal threshold can be expressed as follows,

$$
\gamma_{T}^{*}=\frac{1}{2}\left[Q^{-1}\left(\frac{1}{2}+\frac{1}{2} \sum_{k=0}^{\infty}(-1)^{k+1} \frac{k !}{\alpha^{k}} \frac{(2 k-1) ! !}{(2 k) ! !}\right)\right]^{2}
$$

For large values of $\alpha$, the optimal switching threshold $\gamma_{T}^{*}$ can be approximated, by considering only the first three terms of the infinite series,

$$
\gamma_{T}^{*} \approx \frac{1}{2}\left[Q^{-1}\left(\frac{1}{2}\left(\frac{1}{2 \alpha}-\frac{3}{4 \alpha^{2}}\right)\right)\right]^{2}
$$

A plot of the optimal threshold $\gamma_{T}^{*}$ (in $\mathrm{dB}$ ) against $\alpha$ (in $\mathrm{dB}$ ) is shown in Fig. 2 which shows that the approximate solution to the optimal threshold is very close to the exact one for large values of $\alpha$ greater than $10 \mathrm{~dB}$. Also, the average BER of the secondary receiver is depicted in Fig. 3 for both exact and approximate expressions of $\gamma_{T}^{*}$. It can be clearly seen that the approximate expression of $\gamma_{T}^{*}$ provides almost exact expression to the average BER for values of $\alpha$ greater than 5 dB.

\section{Performance Comparison Between Transmit SSC AND TRANSMIT SC}

In this section, we consider performance evaluation of the transmit selection combining (SC) technique when applied to the secondary transmitter of the UCR system. This combining technique selects the transmit antenna that provides the highest SNR at the secondary receiver. Let $\gamma_{s c}$ denotes the secondary receiver SNR with transmit SC technique applied at the secondary transmitter. Assuming i.i.d transmit diversity branches, the PDF of $\gamma_{s c}$ is expressed as [7]

$$
f_{\gamma_{s c}}\left(\gamma_{s c}\right)=\frac{2 \alpha \gamma_{s c}}{\left(\gamma_{s c}+\alpha\right)^{3}}
$$

The MGF of $\gamma_{s c}$ can be expressed as

$$
\begin{aligned}
M_{\gamma_{s c}}(-p)= & +p \alpha+\left[(p \alpha+1)^{2}-1\right] \\
& \times \mathrm{e}^{(\mathrm{p} \alpha)} \operatorname{Ei}(-\mathrm{p} \alpha), \quad \mathrm{p} \geq 0
\end{aligned}
$$

Similarly, by using the MGF-based approach for the evaluation of the average BER and by using the series expansion of $\operatorname{Ei}(x)$, the average BER for BPSK transmission over a flat Rayleigh fading channel can be expressed as

$$
\begin{aligned}
P_{b}(E ; \alpha) & =\sum_{k=1}^{\infty}(-1)^{k+1} \frac{k !}{\alpha^{k}} \frac{(2 k-1) ! !}{(2 k) ! !} \\
& +\frac{1}{2} \sum_{k=2}^{\infty}(-1)^{k+1} \frac{k !}{\alpha^{k-1}} \frac{(2 k-3) ! !}{(2 k-2) ! !}
\end{aligned}
$$

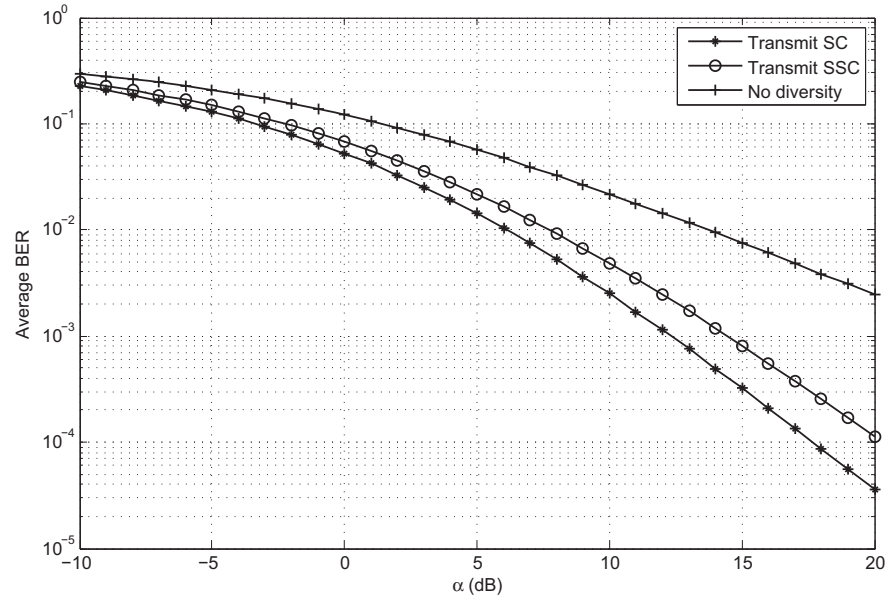

Fig. 4. Average BER comparison between dual branch transmit SSC and dual branch transmit SC in an UCR network versus $\alpha$ in $\mathrm{dB}$ for BPSK transmission over flat Rayleigh channel.

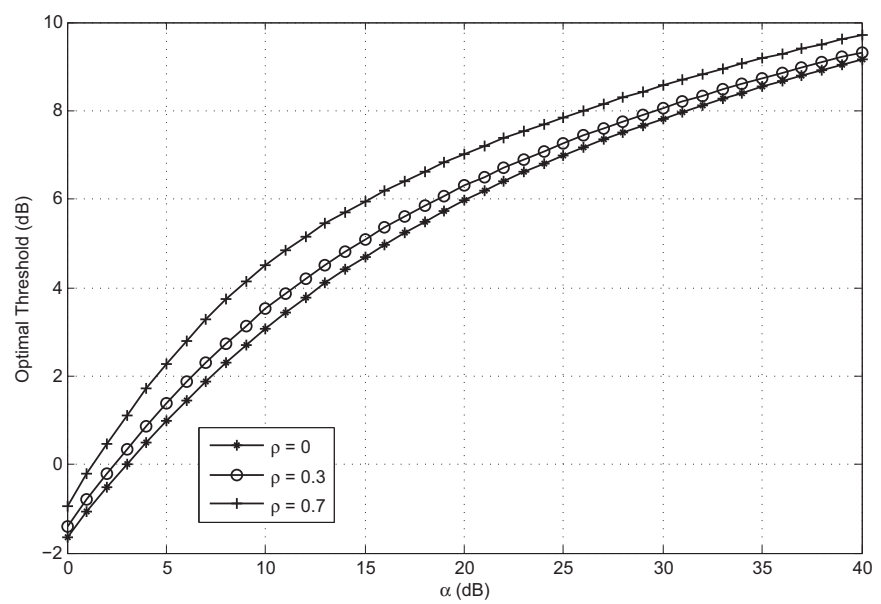

Fig. 5. Transmit SSC optimal switching threshold in $\mathrm{dB}$ for different degrees of correlation between primary and secondary channels versus $\alpha$ in $\mathrm{dB}$ for BPSK transmission over flat Rayleigh channel.

Fig. 4 shows the average BER of dual branch transmit SSC with the optimal switching threshold, dual branch transmit SC and the no-diversity case. Although transmit SSC technique is less efficient in performance than transmit SC technique, it still has a considerable performance gain over the no-diversity case.

\section{EFFect Of Correlation Between Interference And SeCOndary Channels}

In this section, we examine the impact of fading correlation between the secondary channel $g_{s}$ and the interference channel $g_{p}$ on the average BER of the secondary receiver in case of dual branch transmit SSC. When $\sqrt{g_{s}}$ and $\sqrt{g_{p}}$ are correlated Rayleigh random variables with a correlation coefficient $\rho, \gamma_{i}$ would be distributed according to the following pdf [9],

$$
f_{\gamma}(\gamma)=(1-\rho) \frac{\alpha(\gamma+\alpha)}{\left[(\gamma+\alpha)^{2}-4 \rho \alpha \gamma\right]^{3 / 2}}
$$




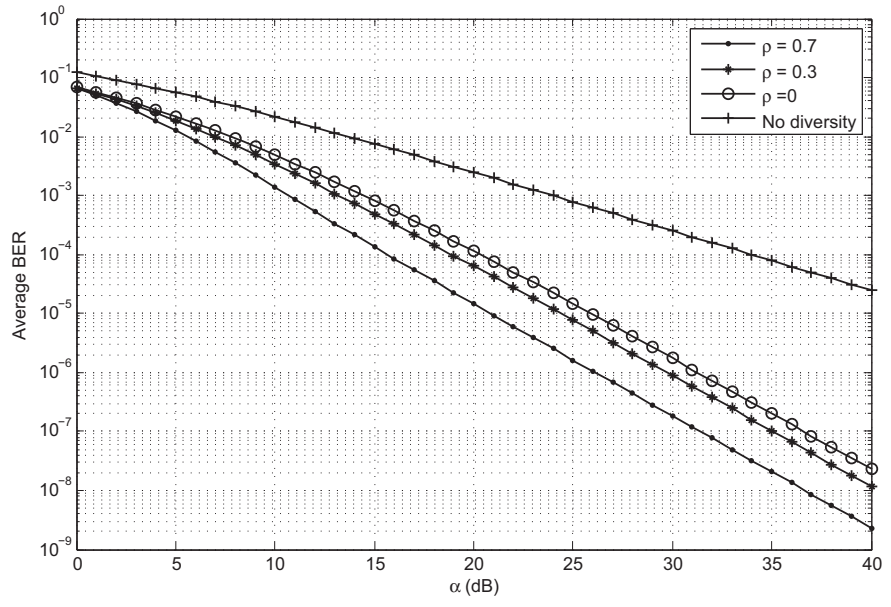

Fig. 6. Transmit SSC average BER for different degrees of correlation between interference and secondary channels versus $\alpha$ in $\mathrm{dB}$ for BPSK transmission over flat Rayleigh channels.

Hence, similarly, the optimal threshold and the average BER can be computed as follows,

$$
\begin{gathered}
\gamma_{T}^{*}=\frac{1}{2}\left[Q^{-1}\left(\frac{1}{2} \int_{0}^{\infty} \operatorname{erfc}(\sqrt{\gamma}) f_{\gamma}(\gamma) d \gamma\right)\right]^{2} \\
P_{b}(E ; \alpha)=\frac{1}{2} F_{\gamma}\left(\gamma_{T}\right) \int_{0}^{\infty} \operatorname{erfc}(\sqrt{\gamma}) f_{\gamma}(\gamma) d \gamma \\
+\int_{\gamma_{T}}^{\infty} \operatorname{erfc}(\sqrt{\gamma}) f_{\gamma}(\gamma) d \gamma
\end{gathered}
$$

The above integrals can be solved numerically to obtain the optimal threshold and the average BER for different degrees of correlation between interference and secondary channels. Fig. 5 and 6 depict the optimal switching threshold in $\mathrm{dB}$ and the average BER respectively versus $\alpha$ in $\mathrm{dB}$ for different values of $\rho$. It follows from Fig. 6 that the average BER of the secondary link decreases as the interference and secondary channels become more correlated. This can be justified by examining that the PDF values defined in (26) shown in Fig. 7 around zero. Based on the fact, that the average BER is dictated by the PDF values close to zero, it is evident from Fig. 7 that as the correlation coefficient increases, the PDF values around zero is getting smaller and hence the average BER is decreasing. Another intuitive justification follows from the fact that $g_{s}$ and $g_{p}$ become equal if $\rho$ is set to 1 and so the channel seen by the secondary receiver, which is the ratio between $\sqrt{g_{s}}$ and $\sqrt{g_{p}}$, reduces to Gaussian channel. Furthermore, as $\rho$ increases, this ratio asymptotically approaches 1 and the channel tends to be Gaussian. Consequently, the average BER of the secondary link decreases with higher degrees of correlation between interference and secondary channels.

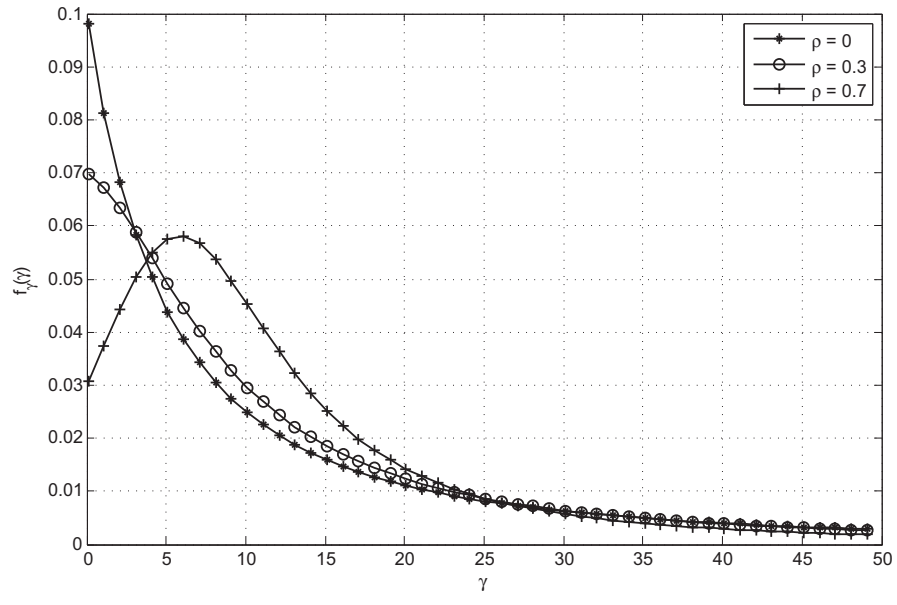

Fig. 7. PDF of the received SNR $\gamma$ defined in (26) for different values of the correlation coefficient $\rho$.

\section{CONCLUSION}

In this paper, we presented a performance analysis of the dual branch transmit SSC technique for UCR system. We showed how to optimize the system performance by calculating the optimal switching threshold that minimizes the average BER of the secondary link. We also derived an expression for the average BER of the dual branch transmit SC technique. We showed that although transmit SSC is less efficient than transmit SC but it still have a considerable diversity gain with the advantage of being more simpler and practical than transmit SC. Finally, we studied the effect of correlation between the interference and the secondary channels on the average BER of the transmit SSC technique showing that the average BER decreases as the correlation between the channels increases.

\section{REFERENCES}

[1] A. Ghasemi and E. Sousa, "Fundamental limits of spectrum-sharing in fading environments," IEEE Trans. on Wireless Comm., vol. 6, no. 2, pp. 649-658, Feb. 2007.

[2] Y. Ko, M.-S. Alouini, and M. Simon, "Analysis and optimization of switched diversity systems," IEEE Trans. on Vehicular Technology, vol. 49, No. 5, pp. 1813-1831, Sep. 2000.

[3] A. Abu-Dayya and N. Beaulieu, "Analysis of switched diversity systems on generalized-fading channels," IEEE Trans. on Comm., vol. 42, pp. 2959-2966, Nov. 1994.

[4] M. Blanco and K. Zdunek, "Performance and optimization of switched diversity systems for the detection of signals with Rayleigh fading," IEEE Trans. on Comm., vol. 27, pp. 1887-1895, Dec. 1979.

[5] G. Stuber, Principles of Mobile Communications. Norwell, MA: Kluwer 1996.

[6] H. Wang, J. Lee, S. Kim and D. Hong, "Capacity enhancement of secondary links through spatial diversity in spectrum sharing," IEEE Trans. on Wireless Comm., vol. 9, no. 2, pp. 494-499, Feb. 2010.

[7] A. Papoulis and S. Pillai, Probability, Random Variables and Stochastic Process. McGraw-Hill, 2002.

[8] I. Gradshteyn and I. Ryzhik, Table of Integrals, Series and Products, 6th ed. San Diego, CA: Academic, 2000.

[9] M. Nakagami, "The m-distribution-A general formula of intensity distribution of rapid fading," pp. 3-36 in Statistical Methods in Radio Wave Propagation, W. C. Hoffman, ed. Oxford: Pergamon Press, 1960. 